University of Nebraska - Lincoln

DigitalCommons@University of Nebraska - Lincoln

2012

\title{
Effect of gadolinium doping on the electronic band structure of europium oxide
}

\author{
Juan Colon Santana \\ University of Nebraska-Lincoln \\ Joonhee Michael An \\ University of Nebraska-Lincoln \\ Ning Wu \\ University of Nebraska-Lincoln \\ Kirill D. Belashchenko \\ University of Nebraska-Lincoln, belashchenko@unl.edu \\ Xianjie Wang \\ University of Wyoming, Laramie \\ See next page for additional authors
}

Follow this and additional works at: https://digitalcommons.unl.edu/physicsdowben

Part of the Physics Commons

Colon Santana, Juan; Michael An, Joonhee; Wu, Ning; Belashchenko, Kirill D.; Wang, Xianjie; Liu, Pan; Tang, Jinke; Losovyj, Yaroslav B.; Yakovkin, I. N.; and Dowben, Peter A., "Effect of gadolinium doping on the electronic band structure of europium oxide" (2012). Peter Dowben Publications. 248.

https://digitalcommons.unl.edu/physicsdowben/248

This Article is brought to you for free and open access by the Research Papers in Physics and Astronomy at DigitalCommons@University of Nebraska - Lincoln. It has been accepted for inclusion in Peter Dowben Publications by an authorized administrator of DigitalCommons@University of Nebraska - Lincoln. 


\section{Authors}

Juan Colon Santana, Joonhee Michael An, Ning Wu, Kirill D. Belashchenko, Xianjie Wang, Pan Liu, Jinke Tang, Yaroslav B. Losovyj, I. N. Yakovkin, and Peter A. Dowben 


\title{
Effect of gadolinium doping on the electronic band structure of europium oxide
}

\author{
Juan A. Colón Santana, ${ }^{1}$ Joonhee M. An, ${ }^{2}$ Ning Wu, ${ }^{2}$ Kirill D. Belashchenko, ${ }^{2}$ Xianjie Wang, ${ }^{3}$ Pan Liu, ${ }^{3}$ Jinke Tang, ${ }^{3}$ \\ Yaroslav Losovyj, ${ }^{4}$ I. N. Yakovkin, ${ }^{5}$ and P. A. Dowben ${ }^{2}$ \\ ${ }^{1}$ Department of Electrical Engineering, W. Scott Engineering Center, North 16th Street, University of Nebraska-Lincoln, \\ Lincoln, Nebraska 68588-0656, USA \\ ${ }^{2}$ Department of Physics and Astronomy and Nebraska Center for Materials and Nanoscience, University of Nebraska-Lincoln, \\ Theodore Jorgensen Hall, 855 North 16th Street, Lincoln, Nebraska 68588-0299, USA \\ ${ }^{3}$ Department of Physics \& Astronomy, University of Wyoming, Laramie, Wyoming 82071, USA \\ ${ }^{4}$ Center for Advanced Microstructures and Devices, Louisiana State University, 6980 Jefferson Highway, Baton Rouge, Louisiana 70806, USA \\ ${ }^{5}$ Institute of Physics, National Academy of Sciences of Ukraine, Prospect Nauki 46, Kyiv 680028, Ukraine
}

(Received 2 August 2011; revised manuscript received 30 November 2011; published 5 January 2012)

\begin{abstract}
High quality films of $\mathrm{EuO}$ and $\mathrm{Eu}_{0.96} \mathrm{Gd}_{0.04} \mathrm{O}$ were grown on $p$-type $\mathrm{Si}(100)$ via pulsed laser deposition. X-raydiffraction results show that the addition of Gd changes the growth texture from [001] to [111]. Angular-resolved photoemission spectroscopy reveals electron pockets around the $X$ points in $\mathrm{Gd}$-doped EuO, indicating that the band gap in $\mathrm{EuO}$ is indirect. Combined photoemission and inverse photoemission measurements show an apparent transition from $n$-type to $p$-type behavior, which is likely due to band bending near the polar (111) surface.
\end{abstract}

DOI: 10.1103/PhysRevB.85.014406

PACS number(s): 75.50.Pp, 75.70.Ak, 79.60.-i

\section{INTRODUCTION}

Europium oxide is a well-known ferromagnetic semiconductor and a candidate for spin filter barrier materials. ${ }^{1,2}$ Stoichiometric EuO has a Curie temperature $\left(T_{\mathrm{C}}\right)$ of $69 \mathrm{~K}$, which is strongly enhanced by electron doping via rare-earth substitution $^{3-8}$ or oxygen vacancies. ${ }^{4,7-11}$ Furthermore, such doping can tune the conductivity of $\mathrm{EuO}$ to match that of silicon. ${ }^{3,12,13}$ Epitaxial or very strongly textured $\mathrm{EuO}(100)$ films can be grown on $\mathrm{Si}(100)$ wafers with a high quality EuO/Si interface. ${ }^{8,14}$

Both oxygen deficiency and Gd doping are expected to introduce $n$-type donors in EuO, but their effects may be somewhat different. While the $T_{\mathrm{C}}$ of $\mathrm{Gd}$-doped unreduced $\mathrm{Eu}_{0.96} \mathrm{Gd}_{0.04} \mathrm{O}$ is $120 \mathrm{~K},{ }^{7,8}$ it increases to $145 \mathrm{~K}$ when the $\mathrm{Gd}$-doped sample is oxygen deficient $\left(\mathrm{Eu}_{0.96} \mathrm{Gd}_{0.04} \mathrm{O}_{1-x}\right){ }^{8}$ For $\mathrm{Eu}_{0.96} \mathrm{Gd}_{0.04} \mathrm{O}$ a $T_{\mathrm{C}}$ as high as $170 \mathrm{~K}$ has been reported. ${ }^{6}$ The combination of $\mathrm{Gd}$ doping and oxygen vacancies or sufficient doping of either brings $\mathrm{Eu}_{0.96} \mathrm{Gd}_{0.04} \mathrm{O}$ across the metal-insulator transition. EuO (and Gd-doped EuO) is a $4 f$ system whose electronic structure consists of two subsystems: a localized $f$ system and an itinerant spd system. ${ }^{15,16}$ Rareearth dopants have large local moments and also provide carriers to populate the bottom of the conduction band.

In the present work, we show that the addition of Gd leads to the filling of electron pockets, revealing the conduction-band minimum at the $X$ point. We also report that the surface of Gd-doped EuO may appear either $p$ type or $n$ type depending on surface preparation. The Gd doping also results in a change of the texture growth from (100) to (111), so that the surface is nominally a polar surface. We argue that this variability may be explained by band bending, which screens the electrostatic field of the polar surface.

\section{EXPERIMENTAL DETAILS}

There are known complications to the growth of $\mathrm{EuO}$ on silicon. Key among the problems is that the presence of a high oxygen partial pressure at the initial stages of the $\mathrm{EuO}$ film growth leads to formation of $\mathrm{Eu}^{3+}$ (indicative of $\mathrm{Eu}_{2} \mathrm{O}_{3}$ ) at the $\mathrm{Si} / \mathrm{EuO}$ interface. ${ }^{3,13}$ Methods for preparing EuO films reported so far include reactive thermal evaporation of $\mathrm{Eu}$ and molecular beam epitaxy (MBE) under ultrahigh vacuum in the presence of oxygen gas. ${ }^{3,6,13,17,18}$ Here we used pulsed laser deposition (PLD) for the growth of EuO and Gd-doped $\mathrm{EuO}$ on $\mathrm{Si}(100)$, shown previously to be viable. ${ }^{8}$ Hydrofluoric acid (HF) and acetone were used to clean the silicon wafers. Before the deposition, the silicon wafers were annealed at $750{ }^{\circ} \mathrm{C}$ in vacuum, at a pressure of $10^{-5}$ Torr of pure $\mathrm{H}_{2}$ gas to reduce the native $\mathrm{SiO}_{2}$ surface layer from the wafers. Both EuO and Gd-doped EuO films were grown on these wafers using PLD at room temperature. The targets used in the PLD process were either $\mathrm{Eu}(99.9 \%)$ metal or a mixture of $\mathrm{Eu}(99.9 \%)$ and $\mathrm{Gd}$ (99.9\%) metals, as described previously. ${ }^{8}$ We chose the Gd substitution level of $4 \%\left(\mathrm{Eu}_{0.96} \mathrm{Gd}_{0.04} \mathrm{O}\right)$, which was reported optimal. ${ }^{6}$

The texture growth orientation for both $\mathrm{EuO}$ and Gd-doped $\mathrm{EuO}$ films grown in this way was determined by $\mathrm{X}$-ray diffraction (XRD) with $\mathrm{Cu}-K_{\alpha}$ radiation obtained using a Philips X'Pert diffractometer. The undoped stoichiometric EuO films adopt the expected rocksalt structure with a lattice constant of $5.131 \AA$ and exhibit (100) growth texture. A strong (200) reflection is seen at the $2 \theta$ angle of $35.3^{\circ}$, as shown in Fig. 1 and reported elsewhere. ${ }^{8}$ The slightly reduced, oxygendeficient EuO film has a lattice constant of $5.106 \AA$ [Fig. 1(a)]. The $\mathrm{Eu}_{0.96} \mathrm{Gd}_{0.04} \mathrm{O}$ films, in contrast, adopts textured growth along the [111] direction, and a strong (111) Bragg peak is seen at the $2 \theta$ angle of $30.3^{\circ}$. This peak yields the lattice spacing of $2.95 \AA$ [Fig. 1(b)] and a slightly larger lattice constant of 5.11 A compared to undoped EuO films. ${ }^{8}$ We find no evidence of Eu metal either as bulk precipitates or at the surface in either XRD or X-ray photoemission in any of our samples.

Combined ultraviolet photoemission (UPS) and inverse photoemission (IPES) spectroscopies were carried out, as has been done elsewhere for both main group element compound semiconductors ${ }^{19-21}$ and other rare-earth oxides. ${ }^{22}$ Some UPS and IPES spectra were taken in a single ultrahigh vacuum chamber to study the placement of both occupied and unoccupied states under the same conditions. The IPES spectra were 


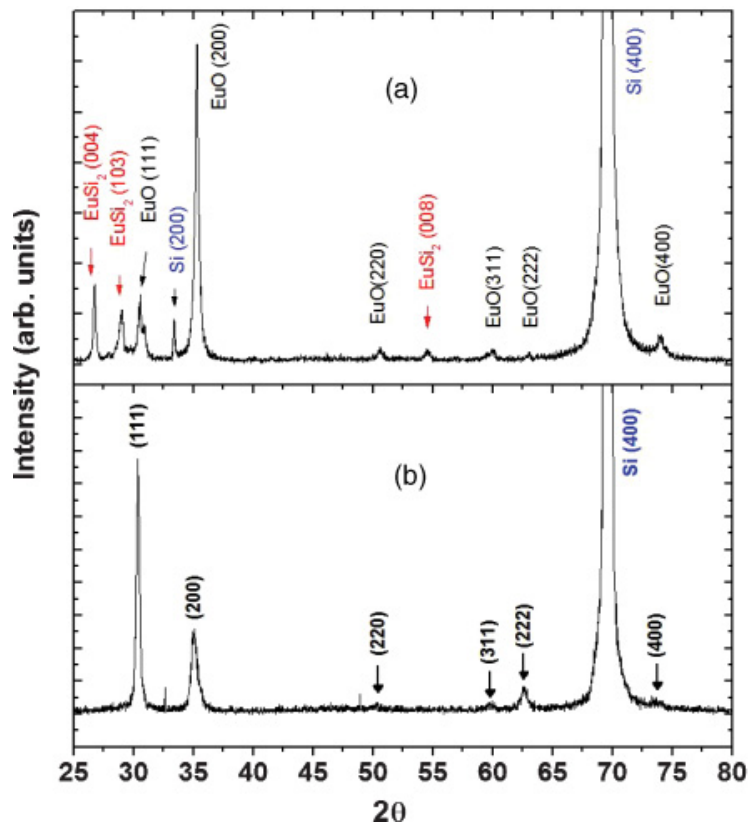

FIG. 1. (Color online) X-ray-diffraction pattern for PLD-grown (a) $\mathrm{EuO}$ and (b) Gd-doped $\mathrm{EuO}$ films on $\mathrm{Si}(100)$.

obtained using variable kinetic-energy incident electrons while detecting the emitted photons at a fixed energy $(9.7 \mathrm{eV})$ with a Geiger-Müller detector. ${ }^{19-24}$ The IPES resolution was limited by an instrumental linewidth of $\sim 400 \mathrm{meV}$, as described elsewhere. ${ }^{23,24}$

The angle-resolved photoemission spectroscopy (ARPES) measurements employed plane-polarized synchrotron radiation dispersed by a $3-\mathrm{m}$ toroidal grating monochromator ${ }^{25,26}$ at the Center for Advanced Microstructures and Devices (CAMD). ${ }^{24}$ The measurements were made in a UHV chamber employing a hemispherical electron analyzer with an angular acceptance of $\pm 1^{\circ}$, as described elsewhere..$^{25,26}$ The combined resolution of the electron energy analyzer and monochromator was $120-150 \mathrm{meV}$ for higher photon energies $(50-120 \mathrm{eV})$.

High-resolution photoemission studies were carried out on the 3-m normal incidence monochromator (NIM) beamline, also at the Center for Advanced Microstructures and Devices (CAMD), ${ }^{27-29}$ as described elsewhere. ${ }^{30,31}$ This normal incidence monochromator is combined with a high-resolution electron energy analyzer (Scienta SES-200) endstation. ${ }^{30}$ The combined resolution (including beamline and analyzer) is 9-15 meV. ${ }^{30}$

In both UPS and IPES measurements the binding energies are referenced with respect to the Fermi edge of copper in intimate contact with the sample surface and reported in terms of $E-E_{\mathrm{F}}$. As described below, the $\mathrm{Eu}_{0.96} \mathrm{Gd}_{0.04} \mathrm{O}$ samples are metallic and exhibit no signs of charging, distinguishing $\mathrm{Eu}_{0.96} \mathrm{Gd}_{0.04} \mathrm{O}$ from the much more dielectric $\mathrm{Gd}_{2} \mathrm{O}_{3}$ and Gd-doped $\mathrm{HfO}_{2}$ grown on $\mathrm{Si}(100){ }^{32}$ For the $\mathrm{Eu}_{0.96} \mathrm{Gd}_{0.04} \mathrm{O}$ samples, the concept of a Fermi level is well defined. For the undoped $\mathrm{EuO}$ the spectra were acquired at elevated temperatures $(\sim 400 \mathrm{~K})$ to suppress residual charging, as is common in the study of dielectric oxide materials of any appreciable thin-film thickness. ${ }^{19-21}$

Segregation and formation of a "surface" $\mathrm{Gd}_{2} \mathrm{O}_{3}$ layer at the surface of the Gd-doped $\mathrm{EuO}$ films can be excluded as the combined photoemission and inverse photoemission are inconsistent with such an insulating (almost certainly $n$-type) surface oxide. ${ }^{33,34}$ The photoemission spectra for $\mathrm{EuO}$ and $\mathrm{Gd}$-doped $\mathrm{EuO}$ differ only slightly. The resonant photoemission intensity is weak, even compared to similarly Gd-doped $\mathrm{HfO}_{2},{ }^{33-35}$ consistent with a more metallic oxide, ${ }^{36}$ and the photovoltaic charging seen with $\mathrm{Gd}_{2} \mathrm{O}_{3}$ and Gd-doped $\mathrm{HfO}_{2}$ (Ref. 32) is not observed here, also consistent with the absence of a dielectric "surface" $\mathrm{Gd}_{2} \mathrm{O}_{3}$ layer.

\section{VALENCE-BAND ELECTRONIC STRUCTURE}

The valence-band photoemission (ARPES) spectra for both doped and undoped EuO films are shown in Fig. 2. These spectra exhibit photoemission features attributable to the Eu $4 f$ states near the Fermi level and the $\mathrm{O} 2 p$ states at about 4-6 eV below the Fermi level. The binding energy of the $\mathrm{O}$ states is consistent with GW calculations, ${ }^{37}$ while LSDA or LSDA $+U$ calculations place the binding energy of the $\mathrm{O}$ states significantly closer to the Fermi level. ${ }^{11,15,16,37-42}$ The binding energies of Eu $4 f$ and oxygen $2 p$ orbitals are also consistent with previous photoemission studies of undoped EuO films. ${ }^{38-45}$ The splitting of the Eu $4 f$ states in the photoemission spectra has been previously reported, ${ }^{42}$ and in those high-resolution photoemission studies, the splitting was clearly resolved at the surface Brillouin-zone center $(\bar{\Gamma})$ for undoped $\mathrm{EuO}(100)$.

The features observed in photoemission spectra are usually wider than the corresponding peaks in the calculated density of states (DOS). The broadening of the photoemission features in the energy range corresponding to the valence band results from various solid-state effects. Strong contributions have been

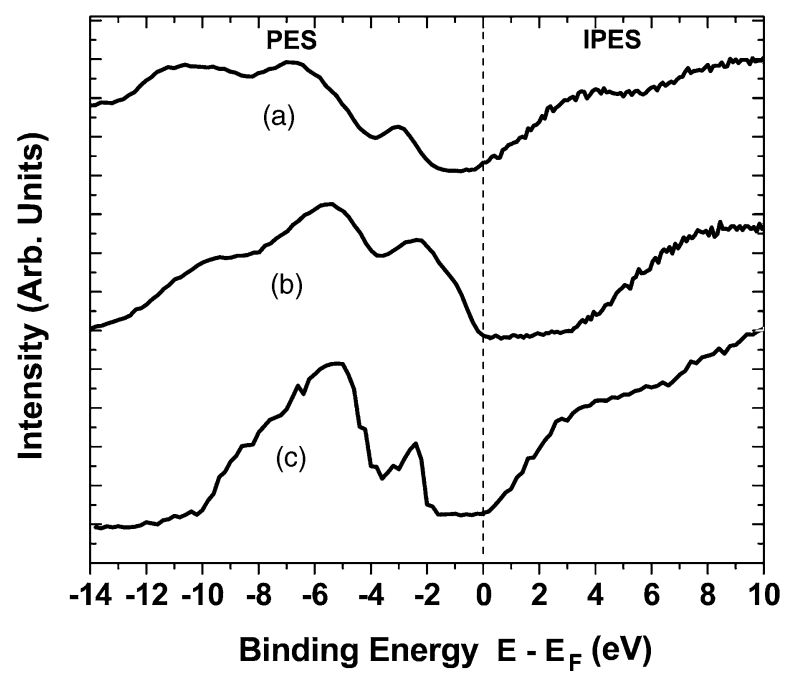

FIG. 2. Combined ARPES and IPES spectra for (a) undoped EuO film, (b) $\mathrm{Eu}_{0.96} \mathrm{Gd}_{0.04} \mathrm{O}$ (111) film, and (c) same as (b) but after sputtering. A photon energy of $60 \mathrm{eV}$ was used, with light incident at $45^{\circ}$ and photoelectrons collected along the surface normal. For the IPES spectra the electrons are incident along the surface normal. 
generally attributed to final-state effects, such as (i) existence of several nearly degenerated excited states; (ii) presence of the OVV Auger peaks; (iii) shake-down satellites of the $5 p$ line ("replicas"). ${ }^{46,47}$ In addition, for a $4 f$ system the relative heights of the $f$ and $d$ photoemission peaks strongly depend on the photon energy, so that for moderate photon energies $(30-50 \mathrm{eV})$, when the cross section for $f$ electrons is relatively small, the height of the $f$-derived peak is also small. Thus there are a number of complications in making a direct comparison of the photoemission spectra with the calculated ground-state DOS. Nonetheless, in most cases the main features of the calculated occupied DOS represent appropriate initial states generating the corresponding peaks in the photoemission spectra.

The broad feature in the photoemission spectra of EuO at binding energies of more than $8 \mathrm{eV}$ below $E_{\mathrm{F}}$ cannot be explained as states originating from $\mathrm{O} 2 p$ orbitals only. There are a number of possible explanations including photoexcitation of $5 s$ or $5 p$ electrons of $\mathrm{Eu}$, which can be followed by the OVV Auger decay leading to the appearance of the $4 f^{6} 5 d^{1} 6 s^{2}$ electronic configuration. Specifically, in the presence of the $p$ hole, the $4 f-5 d$ excitation and then the $5 d-5 p$ Coster-Kronig transition can affect the emission of another $4 f$ electron, which leads to formation of a $2+$ ion with the $4 f^{5} 5 d^{0} 6 s^{2}$ electronic configuration, responsible for the multiplet structure of the photoemission spectra. Screening by itinerant electrons will result in the $4 f^{6} 5 d^{1} 6 s^{2}$ configuration, thus providing the accumulation of excited atoms, which can give rise to the $-8 \mathrm{eV}$ or greater binding energy features in photoemission spectra.

The excited-state spin-polarized DFT calculations were carried out by one of us (I.Y.) with ABINIT code, ${ }^{48}$ using norm-conserving Troullier-Martins pseudopotentials, ${ }^{49}$ with the Perdew-Burke-Ernzerhof (PBE) form of GGA exchangecorrelation functional, ${ }^{50}$ in order to model the actual photoemission spectra. The $f$ states, as well as semicore or shallow core $5 p$ states of Eu were treated as bands. The $\sim 10^{-3}$ Hartree convergence was achieved with the energy cutoff $40 \mathrm{Ha}$ and $4 \times 4 \times 4$ Monkhorst-Pack $^{51}$ set of $k$ points. The DOS were calculated with the tetrahedron method. The estimated lattice constant for fcc EuO (rocksalt structure) was $a=5.24 \AA$, in reasonable $(\sim 2 \%)$ agreement with experimental value $5.131 \AA .^{8,52}$ The formation of the peaks in DOS, induced by excitations, was addressed by using the method of self-consistent band-structure calculations with fixed occupation numbers ${ }^{53,54}$ Specifically, the spin-up and spin-down occupation numbers for each band at each $k$ point are given explicitly and remain unchanged. The fixed occupation numbers prevent interband transitions, which otherwise would unavoidably end with the electron distribution inherent for the ground state, and allows for estimates of excited states by means of routine self-consistent calculations. By adopting appropriate broadening of the DOS features (the widths of the spectral peaks are difficult to estimate rigorously because of unknown lifetimes of the excited states), it is possible to simulate the photoemission spectra, obtaining a reasonable agreement with experiment (Fig. 3).

The normal configuration of the Eu atom is $4 f^{7} 6 s^{2}$, and, according to Hund's rule, all $4 f$ electrons have a spin-up orientation, which leads to a ferromagnetic (FM) ground

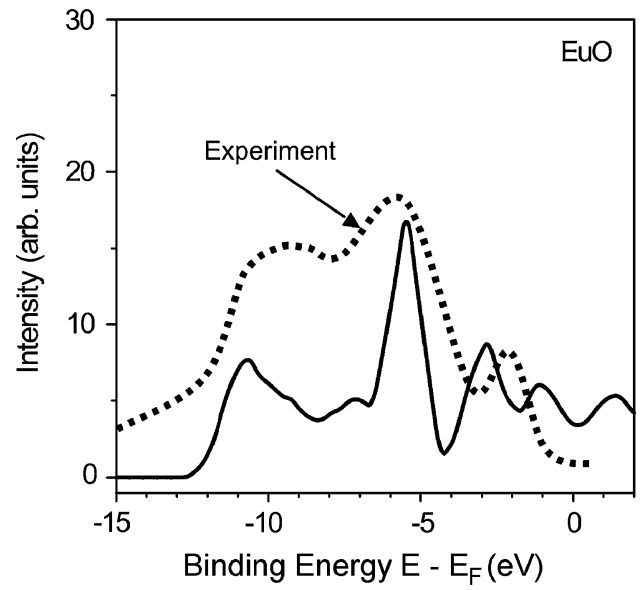

FIG. 3. The simulated (solid line) and experimental (dashed line) photoemission spectra for undoped $\mathrm{EuO}$, with corrections for the combined excited-state density of states. The DFT/GGA calculations of density of states (DOS) were performed with fixed occupancies for treating the excited states.

state also for EuO. Spin-flip transitions like the $4 f \uparrow-5 d \downarrow$ transition in a $\mathrm{Eu}$ atom will result in the formation of the excited state. When the spin-flip transition ends with the occupation of the lowest spin-down band (vacant before the transition), the produced excited state will be metastable due to the spin-conservation rule.

In the excited-state calculation, there are two features that originate from $\mathrm{Eu} 4 f$ states: the one at $1 \mathrm{eV}$ is mostly from the ground-state $\mathrm{Eu} 4 f^{7}$ configuration, while the strong satellite feature is due to excited states as a result of two-hole bound states, which include spin-flip scattering. The feature at about $5.5 \mathrm{eV}$ in Fig. 3 results from the oxygen $\mathrm{O} 2 p$ states, while the features at $7-11 \mathrm{eV}$ stem from a variety of multiconfigurational excited states and satellite features. Thus the peaks in the valence-band photoemission spectra at binding energies of more than $8 \mathrm{eV}$ away from the Fermi level can be explained by photoemission from excited states of EuO. An adroit choice of the pseudopotential, in the excited-state calculation, results in a placement of the $\mathrm{O}$ states with the binding energies in good agreement with experiment.

Although the $\mathrm{Eu}_{0.96} \mathrm{Gd}_{0.04} \mathrm{O}$ films have only a small fraction of $\mathrm{Gd}$ in the lattice, there should be a strong $\mathrm{Gd} 4 f$ contribution at a binding energy of about $-9 \mathrm{eV} .^{33-35,55}$ This contribution becomes evident at photon energies corresponding to the $4 d \rightarrow 4 f$ super-Coster-Kronig resonance, ${ }^{56}$ as in the case of $3 \% \mathrm{Gd}$-doped $\mathrm{HfO}_{2},{ }^{33,35,55}$ but with far less resonant enhancement in the case of $\mathrm{Eu}_{0.96} \mathrm{Gd}_{0.04} \mathrm{O}$. Indeed, the $\mathrm{Gd}$ contribution to the bottom of the valence band, at photon energies off resonance in the case of $\mathrm{Eu}_{0.96} \mathrm{Gd}_{0.04} \mathrm{O}$, is more difficult to discern.

\section{CONDUCTION BAND AND FILLING OF ELECTRON POCKETS}

The unoccupied spectrum in Fig. 2, dominated by Eu $5 d$ states at the conduction-band minimum, is consistent with the reported ${ }^{18}$ oxygen $K$-edge absorption spectrum of EuO. Combined with the angle-resolved core-level XPS (not shown) 


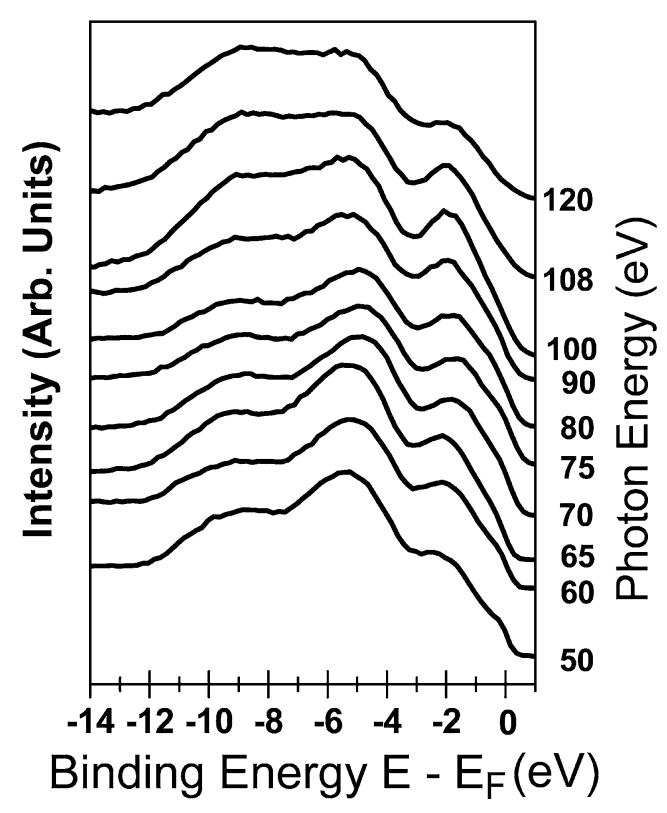

FIG. 4. The photoemission spectra obtained for $\mathrm{Eu}_{0.96} \mathrm{Gd}_{0.04}$ $\mathrm{O}(111)$ films as a function of the photon energy. The angle of incidence was $45^{\circ}$ and all photoelectrons were collected along the surface normal at $T=300 \mathrm{~K}$.

this consistency confirms the stoichiometry of the EuO surface (IPES is extremely surface sensitive).

The electronic band gap for the undoped EuO derived from the combined UPS/ARPES and IPES spectra is about $1.5 \mathrm{eV}$, slightly wider than the optical band gap of 1.1-1.2 eV. ${ }^{57}$ Since EuO has an indirect band gap (see Ref. 37 and below), angleresolved photoemission and IPES, set up in a manner that preferentially samples the center of the surface Brillouin zone, are expected to give a larger band gap corresponding to the band structure at $\bar{\Gamma}$. The placement of the Fermi level close to the conduction-band minimum is consistent with an $n$-type surface, likely due to oxygen vacancies in the lattice.

The main effect of Gd doping is the appearance of new occupied states in the spectrum near the Fermi energy, as seen in Fig. 4. As long as the film is sufficiently crystalline, the ARPES measurements can be used to resolve the spectral intensity by the wave vector component normal to the surface $k_{\perp}$, which can be found as ${ }^{20,21,33,35,58,59}$

$$
k_{\perp}=\sqrt{\frac{2 m}{\hbar^{2}}\left\{E_{\mathrm{kin}} \cos ^{2}(\theta)+U_{\mathrm{in}}\right\}} .
$$

Here $E_{\text {kin }}$ is the photoelectron kinetic energy, $U_{\text {in }}$ is the inner potential, and $\theta$ is the emission angle with respect to the surface normal. Band-structure calculations based on the highly reliable $G W$ method ${ }^{37}$ have shown that the conductionband bottom is at the $X$ point, while the minimum at $\Gamma$ is significantly higher. Therefore we expect that under $4 \% \mathrm{Gd}$ doping the extra carriers will go into the electron pockets at the $X$ points. This expectation was previously confirmed with $2 \%$ cerium doping, ${ }^{60}$ and the present results provide further evidence, as we now explain.

The position of occupied states near the Fermi edge for the $\mathrm{Eu}_{0.96} \mathrm{Gd}_{0.04} \mathrm{O}(111)$ deposited on $\mathrm{Si}(100)$ does show wavevector dependence. By varying the photon energy from 50

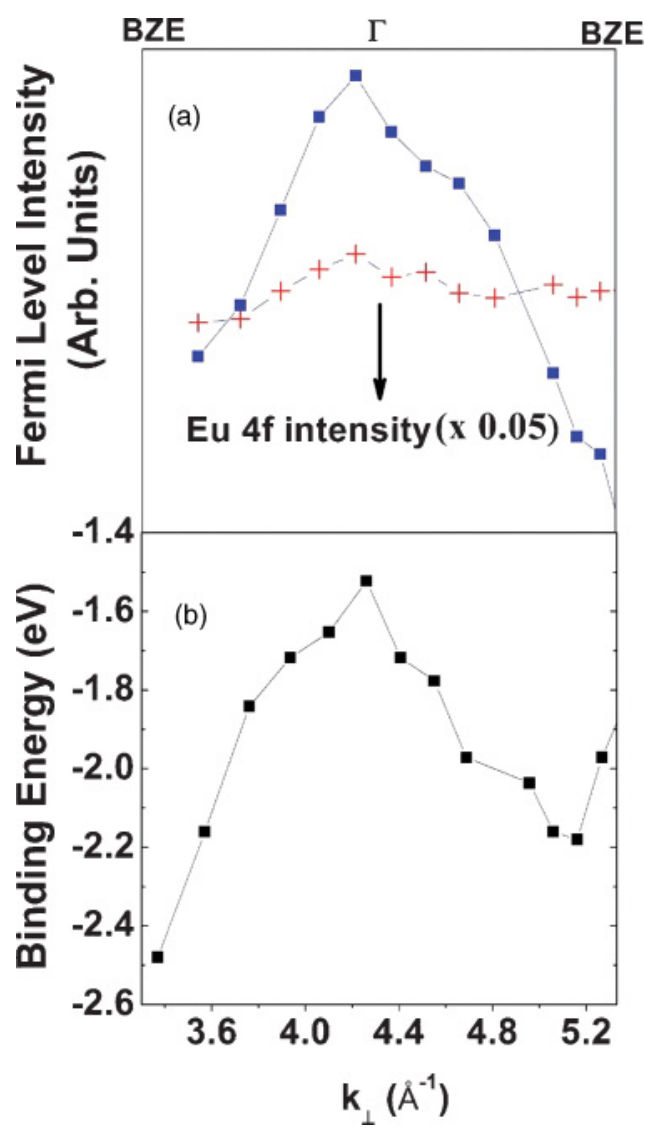

FIG. 5. (Color online) (a) The Fermi level intensity ( $\square$ ) plotted as a function of $k_{\perp}$, along the [111] direction. (b) The dispersion of the Eu $4 f$ weighted band at the valence-band maximum, along the (111) direction or wave vector normal to the surface, $k_{\perp}$. Both results were extracted from the photon energy dependent ARPES spectra. BZE indicates the Brillouin-zone edge. The intensity of the Eu $4 f$ weighted band $(+)$, at the valence-band maximum, along the $k_{\perp}(111)$ direction, is also plotted in (a).

to $158 \mathrm{eV}$, significant dispersion with $k_{\perp}$ (corresponding to the bulk [111] direction) was observed, as seen in Fig. 4. The spectral intensity at the Fermi level, which is plotted in Fig. 5, reaches a maximum at the photon energy corresponding to the bulk $\Gamma$ point. The measured values of $k_{\perp}$ at the bandstructure critical points are consistent with the lattice constants corresponding to the (111) texture determined from XRD. This increase in the intensity at $\Gamma$ near the Fermi level is consistent with the spectral "tail" extending from the states at the top of the valence band, which is at the $\Gamma$ point. This is more evident in the analysis of the band dispersion parallel to the surface, discussed below. This band-structure related effect occurs at photon energies corresponding to the $\Gamma$ points at photon energies well below those that correspond to the $4 d \rightarrow 4 f$ super-Coster-Kronig resonance. ${ }^{56}$

Even though our $\mathrm{Si}(100) / \mathrm{Eu}_{0.96} \mathrm{Gd}_{0.04} \mathrm{O}(111)$ films are not single crystals, they are sufficiently ordered to manifest band dispersion also parallel to the surface along the $\overline{\Gamma M}$ line of the surface Brillouin zone. Indeed, high-resolution photoemission in the transmission mode (of the spectrometer) reveals a variation of the spectral intensity as a function of the parallel 


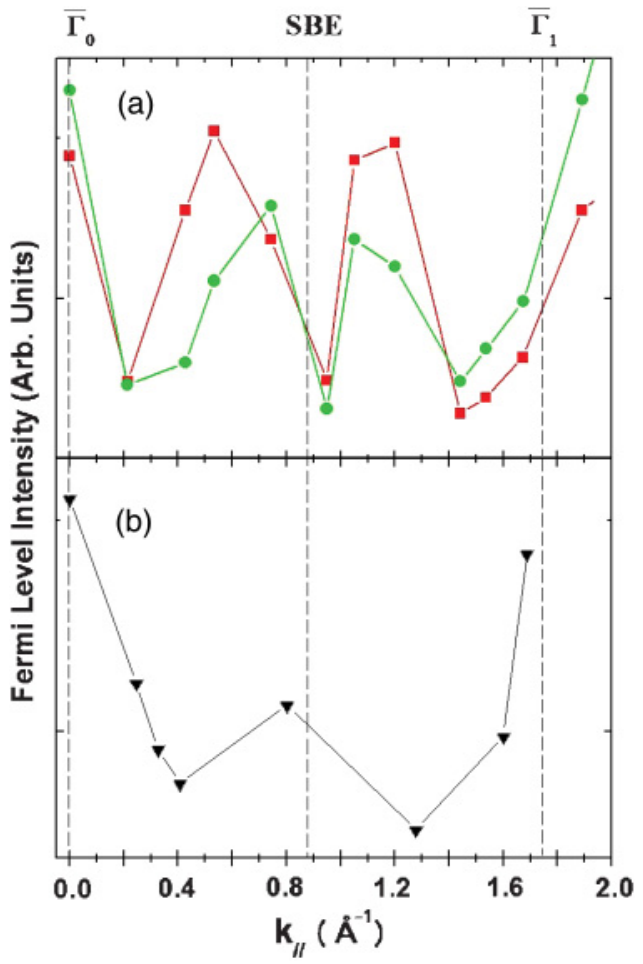

FIG. 6. (Color online) The Fermi-level intensity plotted as a function of the parallel momentum $k_{\|}$along the $\overline{\Gamma \bar{M}}$ direction, for photon energies of (a) $40 \mathrm{eV}$ and (b) $25 \mathrm{eV}$. For $k_{\|}$along the $\overline{\Gamma \mathrm{M}}$ direction, the relative intensity has been also plotted $300-400 \mathrm{meV}$ below the Fermi level (green $\bullet$ ) and at the Fermi level (red $\boldsymbol{\square}$ ), to illustrate the roughly parabolic shape of the electron pocket about the surface Brillouin-zone edge at $40 \mathrm{eV}$.

momentum component $k_{\|}$, which is given by ${ }^{20,21,58,59}$

$$
k_{\|}=\sqrt{\frac{2 m}{\hbar^{2}} E_{\text {kin }}} \sin (\theta)=0.512 \sqrt{E_{\text {kin }}} \sin (\theta)\left\{\AA^{-1}\right\} .
$$

The $k_{\|}$-resolved spectral intensity in the vicinity of the Fermi level is plotted in Fig. 6. The spectral intensity is seen to depend both on the photon energy and on the emission angle $\theta$. This photon energy dependence at the surface Brillouin-zone edge is consistent with the location of the electron pocket at the $X$ point. At 40-eV photon energy the spectral intensity indicates a band crossing on both sides of the surface Brillioun-zone edge. At slightly higher binding energies $(\sim 300 \mathrm{meV}$ below the Fermi level) the intensity maxima move closer to the $X$ point, consistent with the parabolic dispersion of the electron pocket. The fact that the intensities sharply increase at the $\bar{\Gamma}$ points, as we move down from the Fermi level indicates that this intensity represents the spectral tail from the valence-band maximum at $\Gamma$, rather than the conduction-band states. At a photon energy of $25 \mathrm{eV}$ [Fig. 6(b)], corresponding to a lateral shift from the $X$ point along the face of the Brillouin zone, the cut through the bulk band structure has the electron pocket just touching the Fermi level.

\section{BAND BENDING AT THE EuO(111) SURFACE}

In the combined ARPES and IPES spectra shown in Fig. 2 the $\mathrm{Eu}_{0.96} \mathrm{Gd}_{0.04} \mathrm{O}(111)$ surface appears to be $p$ type. At first sight this feature contradicts the observation of filled electron

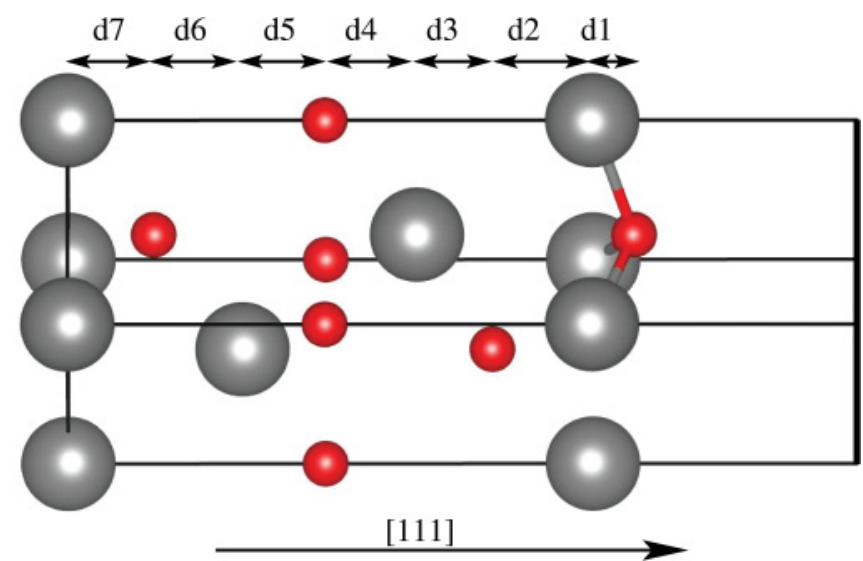

FIG. 7. (Color online) Calculated structure of the O-terminated $\mathrm{EuO}(111)$ surface. Only half of the slab is shown, the other half being symmetric. For interlayer spacings see Table I. In the figure, large spheres are Eu atoms; small spheres: $\mathrm{O}$ atoms.

pockets at the $X$ point and the $n$-type character expected of a material dominated by substitutional Gd dopants and oxygen vacancies, both of which are electron donors. The apparent $p$-type character of this electron-doped sample can only be explained by band bending near the surface, which reveals the presence of a volume charge there. Specifically, the electrostatic potential near the surface is lowered relative to the bulk, which means that the surface itself is negatively charged.

The presence of an uncompensated charge on the (111) surface of a rocksalt oxide is not surprising, because this surface orientation is polar. Normally this orientation is unfavorable, because it requires extensive surface reconstruction or charged defects to screen the electric field in the bulk. However, in the metallic electron-doped sample the free carriers are available to screen the surface charge. It is possible that this screening explains the stabilization of the (111) surface orientation in our Gd-doped EuO samples relative to the (100) orientation preferred by the insulating $\mathrm{EuO}$.

Negative charge of the (111) surface strongly suggests its overoxidation. This is natural for $\mathrm{EuO}$, which in the presence of sufficient oxygen readily oxidizes to $\mathrm{Eu}_{2} \mathrm{O}_{3}$. For undoped $\mathrm{EuO}$ this would result in a mixed $2^{+} / 3^{+}$Eu valence at the surface accompanied by the appearance of unfilled $4 f$ states. Since such unfilled states are absent in the IPES spectrum, we conclude that the band bending does not exceed the bulk band gap, and that the Eu $4 f$ states are kept fully filled by the free carriers introduced by electron doping. To summarize, we argue that the moderately overoxidized, negatively charged surface is accompanied by a positively charged subsurface depletion region. This conclusion is supported by the fact that sputtering of the surface results in a "normal" $n$-type spectroscopic pattern, as seen in Fig. 2(c). This shift can be explained by the removal of some of the excess oxygen from the surface.

Since the atomic structure of the (111) surface has not been determined, it is not possible to perform an accurate theoretical investigation of its electronic properties. However, in order to gain a crude understanding of the effects of overoxidation, we first considered an extreme case of a fully O-terminated 
TABLE I. Calculated interlayer spacings (in $\AA$ ) at the Oterminated $\mathrm{EuO}(111)$ surface, as indicated in Fig. 7.

\begin{tabular}{lc}
\hline \hline Label in Fig. 7 & Interlayer spacing in $(\AA)$ \\
\hline$d_{1}$ & 0.73 \\
$d_{2}$ & 1.75 \\
$d_{3}$ & 1.31 \\
$d_{4}$ & 1.6 \\
$d_{5}$ & 1.44 \\
$d_{6}$ & 1.54 \\
$d_{7}$ & 1.49 \\
\hline \hline
\end{tabular}

Eu (111) surface. We chose a 15-monolayer slab consisting of seven Eu and eight $\mathrm{O}$ monolayers, bisected by a Eu layer, and terminated by $\mathrm{O}$ layers on both surfaces. The equilibrium atomic configuration of this slab was found using the projected augmented wave (PAW) method ${ }^{61,62}$ implemented in the VASP package. ${ }^{63-65}$ For the exchange and correlation potential we used the generalized gradient approximation, ${ }^{50}$ adding the Hubbard $U$ correction $^{66}$ for the Eu $4 f$ orbitals $(U=7.5 \mathrm{eV}$ and $J=0.6 \mathrm{eV}$ ). During the otherwise unrestricted relaxation, the in-plane lattice constants were fixed to their bulk values.

Figure 7 shows the obtained equilibrium configuration of the slab. The lateral positions of the atoms are fixed by symmetry; the interlayer distances indicated in Fig. 7 are listed in Table I. There is a very large inward relaxation of the surface O layer (reducing the interlayer distance by nearly half), along with a significant outward relaxation of the subsurface $\mathrm{Eu}$ layer. Changes in the interlayer spacing propagate a few layers into the bulk, but the interlayer spacing in the middle of the slab already deviates by less than $1 \%$ from its bulk value. Such oscillatory interlayer spacings are common for polar oxide surfaces. ${ }^{67}$

To illustrate our interpretation of the band bending near the overoxidized surface, we considered a somewhat arbitrary, but nonetheless suggestive model, using the tight-binding linear muffin-tin orbital (TB-LMTO) method. A 27-monolayer slab was chosen for this purpose, inserting bulklike EuO layers in the middle of the relaxed 15-monolayer slab shown in Fig. 7. The parameters of the calculation, such as the atomic sphere radii, the auxiliary empty spheres, and the $U$ and $J$ parameters were adjusted to reproduce the correct band structure of bulk EuO, including the band gap and the character of the conduction-band states. We also added a fictitious external potential of $1.36 \mathrm{eV}$ to the oxygen sites in order to push the $\mathrm{O} 2 p$ states to lower energies. The effect of Gd doping was included in the virtual crystal approximation (VCA), replacing Eu with fictitious atoms with nuclear charge 63.17 e (corresponding to $17 \% \mathrm{Gd}$ doping). Even at this large doping level the negative charge on the
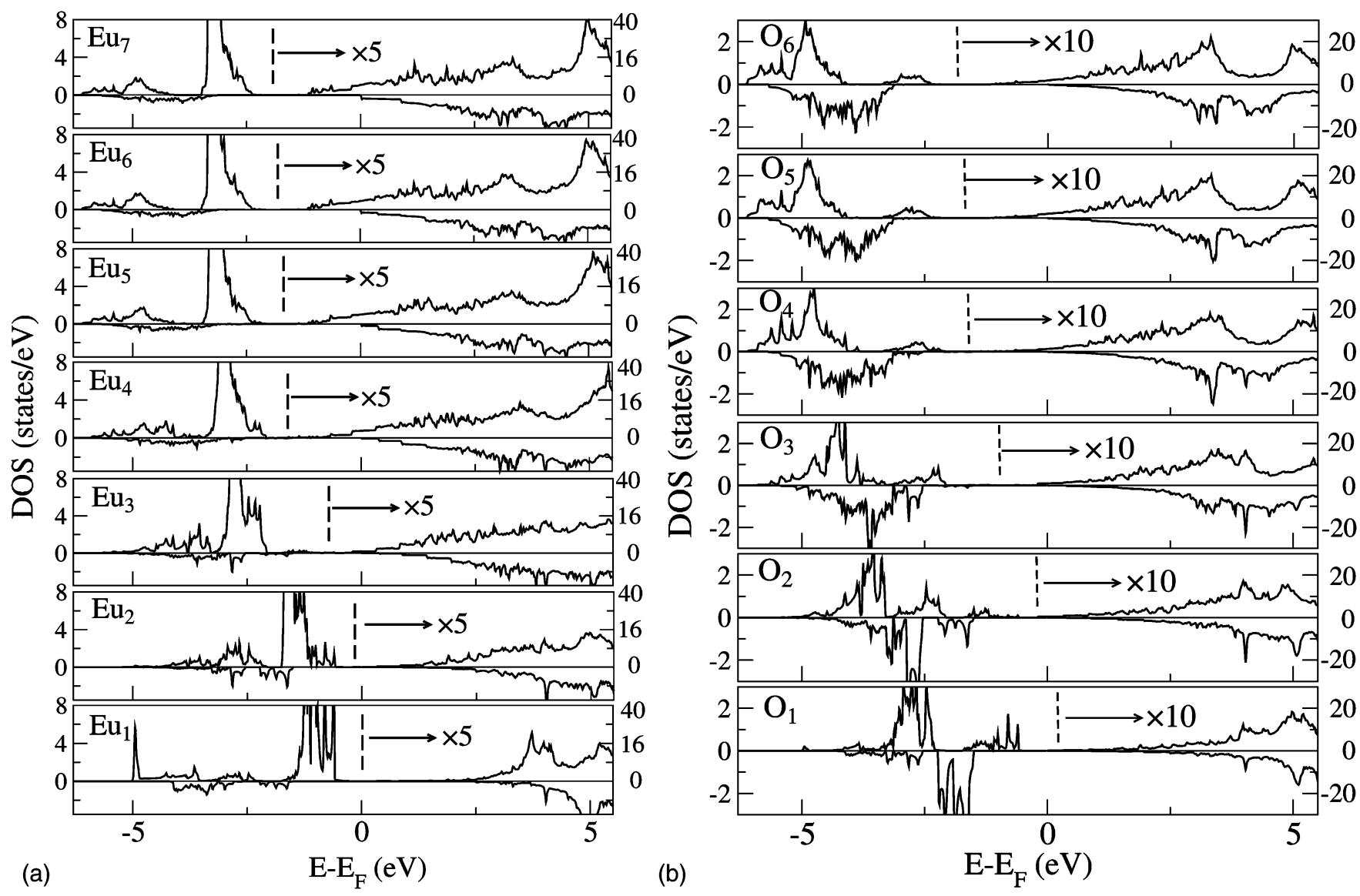

FIG. 8. Site-projected DOS for all Eu and O atoms of the 27-monolayer slab of Gd-doped-EuO with (111) surfaces. A model with empirical adjustments is used (see text). (a) Eu site-projected DOS where the unoccupied DOS is multiplied by 5 (as indicated). (b) O site-projected DOS; unoccupied DOS is multiplied by 10 (as indicated). The numbering of the sites starts at the surface. 


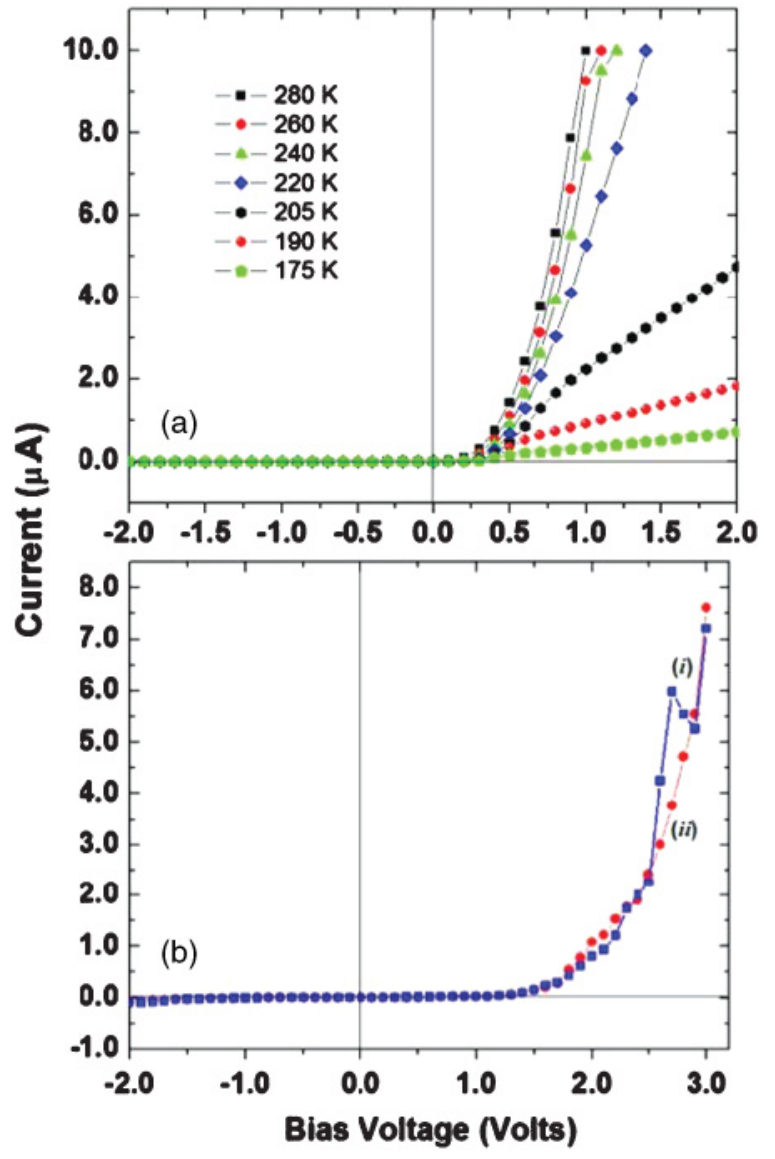

FIG. 9. (Color online) $I-V$ curves for (a) undoped $\mathrm{EuO}$ (100) film as a function of temperature, (b) $\mathrm{Eu}_{0.96} \mathrm{Gd}_{0.04} \mathrm{O}(111)$ film at room temperature. The curve marked $(i)$ is for zero field; the curve marked (ii) is for an external magnetic field of $1000 \mathrm{Oe}$

O-terminated surface is unrealistically large and results in mixed-valent $\mathrm{Eu}$ at the surface. It is clear that the actual amount of surface overoxidation in our experimental samples is much lower. In order to make our illustration more realistic without considering more complicated surface terminations, we reduced the amount of charge transfer to the surface $\mathrm{O}$ layer by replacing the oxygen atoms in this layer by fictitious atoms with nuclear charge $8.40 e$. The partial DOS for all Eu and $\mathrm{O}$ atoms of this slab are shown in Fig. 8. The upward band bending near the surface is clearly seen, which results in a structure similar to a $p-n$ junction with a depletion region.

There is another indication of the band bending of the kind illustrated by Fig. 8. Evidence of the electron pockets at the Fermi level and absence of photovoltaic charging indicate that Gd-doped EuO is metallic. The energy-band diagram with a narrow depletion region suggests that, with a suitable interface, the surface region may operate as a tunnel (Esaki) diode with the current flowing perpendicular to the surface (or to the interface with silicon, assuming similar band bending there). This independent signature of surface band bending in the $\mathrm{Eu}_{0.96} \mathrm{Gd}_{0.04} \mathrm{O}(111)$ sample was indeed observed, as seen in Fig. 9. Although very far from ideal, the $I-V$ curve shows a region of negative differential resistance close to a $3.0-\mathrm{V}$ bias. Interestingly, the application of a magnetic field eliminates this region of negative differential resistance [see Fig. 9(b)]. The origin of this effect is not clear, but it may be mediated by the induction of a small exchange splitting in the conduction band through partial ordering of Eu local moments induced by the magnetic field. These effects are not seen with undoped $\mathrm{EuO}(100)$ thin films on $p$-type silicon, as indicated in Fig. 9(a) for comparison, and thus the (111) texture growth and $\mathrm{Gd}$ (or similar) doping may be required for tunnel (Esaki) diode behavior. It should be noted that $\mathrm{Gd}_{2} \mathrm{O}_{3}$ is typically $n$-type in thin-film form and forms a heterojunction diode with $p$-type $\mathrm{Si}(100)$, but no negative differential resistance has been observed with such structures. ${ }^{33}$ Heterostructures of $\mathrm{Gd}_{2} \mathrm{O}_{3}$ with $\mathrm{Si}(100)$ show diode characteristics ${ }^{19}$ more similar to undoped $\mathrm{EuO} / \mathrm{Si}(100)$ heterostructure [Fig. 9(a)] than those observed with $\mathrm{Eu}_{0.96} \mathrm{Gd}_{0.04} \mathrm{O}(111) / \mathrm{Si}(100)$ heterostructure [Fig. 9(b)].

\section{CONCLUSIONS}

We have found that $\mathrm{Gd}$ doping of $\mathrm{EuO}$ at the level of $4 \%$ changes the texture orientation for PLD-grown films on $\mathrm{Si}(100)$ from (100) to (111). The observation of electron pockets filled under Gd doping confirms the indirect character of the EuO band gap with the conduction-band minima at the $X$ points, in agreement with GW calculations ${ }^{37}$ and prior data for Ce-doped EuO. ${ }^{60}$ The polar character of the (111) surface results in a significant band bending at the surface, which appears to depend on the degree of the surface overoxidation. An $I-V$ characteristic reminiscent of an Esaki diode behavior further suggests the existence of a depletion region in the subsurface region, which is associated with this band bending.

\section{ACKNOWLEDGMENTS}

The authors acknowledge insightful discussions with Andre Petukhov, W. Nolting, and W. Borgiel. The authors would like to thank Jie Xiao for his technical help with some of the measurements. This work was supported by the Defense Threat Reduction Agency (Grant No. HDTRA1-07-1-0008), by NSF through the Nebraska MRSEC (Grant No. DMR-0820521) and Grant No. DMR-0852862. Additional support was provided by the Nebraska Research Initiative. Part of this work was performed at the Center of Advanced Microstructures and Devices, supported by the Louisiana Board of Regents.
${ }^{1}$ T. S. Santos and J. S. Moodera, Phys. Rev. B 69, 241203 (2004).

${ }^{2}$ J. S. Moodera, T. S. Santos, and T. Nagahama, J. Phys.: Condens. Matter 19, 165202 (2007).

${ }^{3}$ A. Schmehl, V. Vaithyanathan, A. Herrnberger, S. Thiel, C. Richter, M. Liberati, T. Heeg, M. Röckerath, L. F. Kourkoutis, S. Mühlbauer,
P. Böni, D. A. Muller, Y. Barash, J. Schubert, Y. Idzerda, J. Mannhart, and D. G. Schlom, Nat. Mater. 6, 882 (2007).

${ }^{4}$ T. Matsumoto, K. Yamaguchi, M. Yuri, K. Kawaguchi, N. Koshizaki, and K. Yamada, J. Phys.: Condens. Matter 16, 6017 (2004).

${ }^{5}$ M. W. Shafer and T. R. McGuire, J. Appl. Phys. 39, 588 (1968). 
${ }^{6}$ H. Ott, S. J. Heise, R. Sutarto, Z. Hu, C. F. Chang, H. H. Hsieh, H.-J. Lin, C. T. Chen, and L. H. Tjeng, Phys. Rev. B 73, 094407 (2006).

${ }^{7}$ T. Mairoser, A. Schmehl, A. Melville, T. Heeg, L. Canella, P. Böni, W. Zander, J. Schubert, D. E. Shai, E. J. Monkman, K. M. Shen, D. G. Schlom, and J. Mannhart, Phys. Rev. Lett. 105, 257206 (2010). ${ }^{8}$ X. Wang, P. Liu, K. A. Fox, J. Tang, J. A. Colón Santana, K. Belashchenko, P. A. Dowben, and Yu Sui, IEEE Trans. Magn. 46, 1879 (2010).

${ }^{9}$ A. S. Borukhovich, V. G. Bamburov, and A. A. Sidorov, J. Magn. Magn. Mater. 73, 106 (1988).

${ }^{10}$ O. Massenet, Y. Capoimont, and N. Van Dang, J. Appl. Phys. 45, 3593 (1974)

${ }^{11}$ M. Barbagallo, N. D. M. Hine, J. F. K. Cooper, N.-J. Steinke, A. Ionescu, C. H. W. Barnes, C. J. Kinane, R. M. Dalgliesh, T. R. Charlton, and S. Langridge, Phys. Rev. B 81, 235216 (2010).

${ }^{12}$ M. R. Oliver, J. O. Dimmock, A. L. McWhorter, and T. B. Reed, Phys. Rev. B 5, 1078 (1972).

${ }^{13}$ R. P. Panguluri, T. S. Santos, E. Negusse, J. Dvorak, Y. Idzerda, J. S. Moodera, and B. Nadgorny, Phys. Rev. B 78, 125307 (2008).

${ }^{14}$ J. Lettieri, V. Vaithyanathan, S. K. Eah, J. Stephens, V. Sih, D. D. Awschalom, J. Levy, and D. G. Schlom, Appl. Phys. Lett. 83, 975 (2003).

${ }^{15}$ P. Larson and W. R. L. Lambrecht, J. Phys.: Condens. Matter 18, 11333 (2006).

${ }^{16}$ R. Schiller and W. Nolting, Solid State Commun. 118, 173 (2001).

${ }^{17}$ K. Sattler and H. C. Siegmann, Phys. Rev. Lett. 29, 1565 (1972).

${ }^{18}$ P. G. Steeneken, L. H. Tjeng, I. Elfimov, G. A. Sawatzky, G. Ghiringhelli, N. B. Brookes, and D.-J. Huang, Phys. Rev. Lett. 88, 047201 (2002).

${ }^{19}$ I. Ketsman, D. Wooten, Jie Xiao, Ya. B. Losovyj, Ya. V. Burak, V. T. Adamiv, A. Sokolov, J. Petrosky, J. McClory, and P. A. Dowben, Phys. Lett. A 374, 891 (2010).

${ }^{20}$ D. Wooten, I. Ketsman, J. Xiao, Ya. B. Losovyj, J. Petrosky, J. McClory, Ya. V. Burak, V. T. Adamiv, J. M. Brown, and P. A. Dowben, Eur. Phys. J. Appl. Phys. 52, 31601 (2010).

${ }^{21}$ V. T. Adamiv, Ya. V. Burak, D. Wooten, J. McClory, J. Petrosky, I. Ketsman, Jie Xiao, Ya. B. Losovyj, and P. A. Dowben, Materials 3, 4550 (2010).

${ }^{22}$ S. Sayan, T. Emge, E. Garfunkel, Xinyuan Zhao, L. Wielunski, R. A. Bartynski, D. Vanderbilt, J. S. Suehle, S. Suzer, and M. Banaszak-Holl, J. Appl. Phys. 96, 7485 (2004).

${ }^{23}$ J. Zhang, D. N. McIlroy, P. A. Dowben, H. Zeng, G. Vidali, D. Heskett, and M. Onellion, J. Phys.: Condens. Matter 7, 7185 (1995).

${ }^{24}$ D. N. McIlroy, J. Zhang, P. A. Dowben, and D. Heskett, Mater. Sci. Eng. A 217/218, 64 (1996).

${ }^{25}$ Y. Losovyj, I. Ketsman, E. Morikawa, Z. Wang, J. Tang, and P. A. Dowben, Nucl. Instrum. Methods Phys. Res., Sect. A 582, 264 (2007).

${ }^{26}$ P. A. Dowben, D. LaGraffe, and M. Onellion, J. Phys.: Condens. Matter 1, 6571 (1989).

${ }^{27}$ J. Hormes, J. D. Scott, and V. P. Suller, Synchrotron Radiation News 19, 27 (2006).

${ }^{28}$ A. Roy, E. Morikawa, H. Bellamy, C. Kumar, J. Goettert, V. Suller, K. Morris, D. Ederer, and J. Scott, Nucl. Instrum. Methods Phys. Res., Sect. A 582, 22 (2007).

${ }^{29}$ E. Morikawa, J. D. Scott, J. Goettert, G. Aigeldinger, C. S. S. R. Kumar, B. C. Craft, P. T. Sprunger, R. C. Tittsworth, and F. J. Hormes, Rev. Sci. Instrum. 73, 1680 (2002).
${ }^{30}$ Y. Losovyj, K. Morris, L. Rosa, J. D. Scott, and P. A. Dowben, Nucl. Instrum. Methods Phys. Res., Sect. A 582, 258 (2007).

${ }^{31}$ C. M. Evans, J. D. Scott, and E. Morikawa, Rev. Sci. Instrum. 73, 1557 (2002).

${ }^{32}$ D. Wooten, Ya. B. Losovyj, J. Petrosky, J. McClory, J. Tang, W. Wang, and P. A. Dowben, in Rare-Earth Doping of Advanced Materials for Photonic Applications, Edited by V. Dierolf, Y. Fujiwara, U. Hommerich, P. Ruterana, and J. Zavada, MRS Symposium Proceedings No. 1111 (Materials Research Society, Warrendale, 2009), p. D07.

${ }^{33}$ Ya. B. Losovyj, D. Wooten, J. Colon Santana, J. M. An, K. D. Belashchenko, N. Lozova, J. Petrosky, A. Sokolov, J. Tang, W. Wang, N. Arulsamy, and P. A. Dowben, J. Phys.: Condens. Matter 21, 045602 (2009).

${ }^{34}$ T. Komesu, H. K. Jeong, David Wooten, Ya. B. Losovyj, J. N. Crain, M. Bissen, F. J. Himpsel, J. Petrosky, J. Tang, W. Wang, I. N. Yakovkin, and P. A. Dowben, Phys. Status Solidi B 246, 975 (2009).

${ }^{35}$ I. Ketsman, Ya. B. Losovyj, A. Sokolov, Jinke Tang, Zhenjun Wang, K. D. Belashchenko, and P. A. Dowben, Appl. Phys. A 89, 489 (2007).

${ }^{36}$ P. A. Dowben, Surf. Sci. Rep. 40, 151 (2000).

${ }^{37}$ J. M. An, S. V. Barabash, V. Ozolins, M. van Schilfgaarde, and K. D. Belashchenko, Phys. Rev. B 83, 064105 (2011).

${ }^{38}$ J. Kunes, W. Ku, and W. P. Pickett, J. Phys. Soc. Jpn. 74, 1408 (2005).

${ }^{39}$ D. B. Ghosh, M. De, and S. K. De, Phys. Rev. B 70, 115211 (2004).

${ }^{40}$ N. J. C. Ingle and I. S. Elfimov, Phys. Rev. B 77, 121202(R) (2008).

${ }^{41}$ R. Schiller, O. Myrasov, A. J. Freeman, and W. Nolting, J. Magn. Magn. Mater. 226, 388 (2001).

${ }^{42}$ H. Miyazaki, T. Ito, H. J. Im, S. Yagi, M. Kato, K. Soda, and S. Kimura, Phys. Rev. Lett. 102, 227203 (2009).

${ }^{43}$ H. Miyazaki, T. Ito, S. Ota, H. J. Im, S. Yagi, M. Kato, K. Soda, and S.-I. Kimura, Physica B 403, 917 (2008).

${ }^{44}$ P. Munz, Helv. Phys. Acta 49, 281 (1976).

${ }^{45}$ D. E. Eastman, F. Holtzberg, and S. Methfessel, Phys. Rev. Lett. 23, 226 (1969).

${ }^{46}$ S. Haffner, C. G. Olson, and D. W. Lynch, Phys. Rev. B 60, 16346 (1999).

${ }^{47}$ D. M. Wieliczka, C. G. Olson, and D. W. Lynch, Phys. Rev. Lett. 52, 2180 (1984).

${ }^{48}$ X. Gonze, J.-M. Beuken, R. Caracas, F. Detraux, M. Fuchs, G.-M. Rignanese, L. Sindic, M. Verstraete, G. Zerah, F. Jollet, M. Torrent, A. Roy, M. Mikami, Ph. Ghosez, J.-Y. Raty, and D. C. Allan, Comput. Mater. Sci. 25, 478 (2002).

${ }^{49}$ N. Troullier and J. L. Martins, Phys. Rev. B 43, 1993 (1991).

${ }^{50}$ J. P. Perdew, K. Burke, and M. Ernzerhof, Phys. Rev. Lett. 77, 3865 (1996).

${ }^{51}$ H. J. Monkhorst and J. D. Pack, Phys. Rev. B 13, 5188 (1976).

${ }^{52} \mathrm{P}$. Wachter, in Handbook on the Physics and Chemistry of Rare Earths, edited by K. A. Gschneidner and L. Eyring (Elsevier, Amsterdam, 1979), Vol. 2.

${ }^{53}$ N. Wu, D. LaGraffe, I. N. Yakovkin, and P. A. Dowben, Phys. Status Solidi B 248, 1253 (2011).

${ }^{54}$ I. N. Yakovkin, Appl. Surf. Sci. 256, 4845 (2010).

${ }^{55}$ Ya. B. Losovyj, I. Ketsman, A. Sokolov, K. D. Belashchenko, P. A. Dowben, J. Tang, and Z. Wang, Appl. Phys. Lett. 91, 132908 (2007). 
${ }^{56}$ Juan A. Colón Santana, S. McHale, S. R. McHale, J. W. McClory, J. C. Petrosky, J. Wu, A. Rivera, R. Palai, X. Wang, P. Liu, Jinke Tang, Yaroslav Losovyj, and P. A. Dowben (unpublished).

${ }^{57}$ J. O. Dimmock, IBM J. Res. Dev. 314, 301 (1970).

${ }^{58}$ E. W. Plummer and W. Eberhardt, Adv. Chem. Phys. 49, 533 (1982).

${ }^{59}$ N. V. Richardson and A. M. Bradshaw, in Electron Spectroscopy: Theory, Techniques and Applications, edited by C. R. Brundle and A. D. Baker (Academic, San Diego, 1984), Vol. 4, p. 153.

${ }^{60}$ P. Liu, J. Tang, J. A. Colón Santana, K. Belashchenko, and P. A. Dowben, J. Appl. Phys. 109, 07C311 (2011).
${ }^{61}$ P. E. Blöchl, Phys. Rev. B 50, 17953 (1994).

${ }^{62}$ G. Kresse and D. Joubert, Phys. Rev. B 59, 1758 (1999).

${ }^{63}$ G. Kresse and J. Hafner, Phys. Rev. B 48, 13115 (1993).

${ }^{64}$ G. Kresse and J. Furthmüller, Comput. Mater. Sci. 6, 15 (1996).

${ }^{65}$ G. Kresse and J. Furthmüller, Phys. Rev. B 54, 11169 (1996).

${ }^{66}$ A. I. Liechtenstein, V. I. Anisimov, and J. Zaanen, Phys. Rev. B 52, R5467 (1995).

${ }^{67}$ C. Noguera, J. Phys.: Condens. Matter 12, R367 (2000). 